\title{
A simple nearest-neighbor two-body Hamiltonian system for which the ground state is a universal resource for quantum computation
}

\author{
Stephen D. Bartlett ${ }^{1}$ and Terry Rudolph ${ }^{2,3}$ \\ ${ }^{1}$ School of Physics, The University of Sydney, Sydney, New South Wales 2006, Australia \\ ${ }^{2}$ Department of Physics, Imperial College London, London SW7 2BW, United Kingdom \\ ${ }^{3}$ Institute for Mathematical Sciences, Imperial College London, London SW7 2BW, United Kingdom
}

(Dated: 24 October 2006)

\begin{abstract}
We present a simple quantum many-body system - a two-dimensional lattice of qubits with a Hamiltonian composed of nearest-neighbor two-body interactions - such that the ground state is a universal resource for quantum computation using single-qubit measurements. This ground state approximates a cluster state that is encoded into a larger number of physical qubits. The Hamiltonian we use is motivated by the projected entangled pair states, which provide a transparent mechanism to produce such approximate encoded cluster states on square or other lattice structures (as well as a variety of other quantum states) as the ground state. We show that the error in this approximation takes the form of independent errors on bonds occurring with a fixed probability. The energy gap of such a system, which in part determines its usefulness for quantum computation, is shown to be independent of the size of the lattice. In addition, we show that the scaling of this energy gap in terms of the coupling constants of the Hamiltonian is directly determined by the lattice geometry. As a result, the approximate encoded cluster state obtained on a hexagonal lattice (a resource that is also universal for quantum computation) can be shown to have a larger energy gap than one on a square lattice with an equivalent Hamiltonian.
\end{abstract}

PACS numbers: 03.67.Lx

The state of a quantum many-body system can serve as a universal resource for quantum computing, where computation proceeds through single-qubit measurements alone [1, 2, 3]. This observation raises the intriguing possibility that there exist physical systems which are "naturally" quantum computers. More precisely, one may ask whether there exist quantum many-body systems which, when cooled sufficiently close to the ground state, can be used for quantum computation by simply making individual measurements on the constituent particles.

It is straightforward to write down a Hamiltonian for a many-body spin system for which this is the case - one needs only to take the (negative) sum of the stabilizer operators corresponding to, say, a square lattice cluster state [4]. This Hamiltonian is somewhat unsatisfactory, however, because it involves five-body interactions, and fundamental interactions are strictly between two bodies. Using the results of Haselgrove et al. [5], Nielsen proved the following negative result: a cluster state suitable for quantum computation cannot arise as the exact ground state of any Hamiltonian involving only local twobody interactions [3]. However, Oliveira and Terhal 6], building on the results of Kempe, Kitaev and Regev [7], demonstrated that cluster states (and other such states that are universal) can be approximated by the ground state of a local two-body Hamiltonian. The key idea of their result is to make use of "mediating" ancilla qubits to create an effective many-body coupling out of two-body interactions.

Here, we provide a simple and explicit scheme for cluster-state quantum computation using the ground state of a Hamiltonian consisting of only two-body inter- actions. Our approach, which provides an alternative to the use of mediating systems as in [6, 7], is motivated by the projected entangled pair states (PEPS) as proposed by Verstraete and Cirac [8]. Using PEPS, it was demonstrated that the cluster state can be obtained through a projection of a number of virtual qubits prepared in maximally-entangled states down to a lesser number of physical qubits. We turn this construction around, and consider how, through the use of nearest-neighbor twobody Hamiltonians, we may effect a similar projection but in this instance of a number of physical qubits down to a lesser number of logical qubits. The system we investigate is gapped, as the energy difference between the ground and first-excited states is independent of the size of the lattice. The low-energy theory is described by an effective five-body interaction Hamiltonian that is precisely equal to the (negative) sum of the stabilizer operators acting on the logical qubits. That is, a cluster state encoded as a logical state is approximated by the ground state of a larger number of physical qubits. This encoding provides a very transparent mechanism for achieving the effective many-body coupling out of two-body interactions, which can be of Ising and Heisenberg form, and is naturally extendible to other PEPS constructions. Crucially, despite using logical qubits consisting of several physical qubits, measurement-based quantum computation can proceed on the encoded cluster state using only single-qubit measurements.

We also investigate the usefulness of this ground state for measurement-based quantum computation. Specifically, we analyze the errors associated with the fact that the ground state only approximates the encoded cluster 
state, and demonstrate that the (fixed) energy gap is directly determined by the lattice geometry.

The PEPS on a square lattice 8] consists of a 2dimensional lattice of virtual qubits following the Archimedian tiling 4.8.8 (also known as a "CaVO" lattice) [9], as shown in Fig. 1 Quadruples of virtual qubits on the vertices of the 4 -vertex tiles (such as those circled) form sites. Pairs of virtual qubits at neighboring sites, connected by dashed-line bonds in Fig. 1. are prepared in the two-qubit cluster state 21]

$$
\left|C_{2}\right\rangle=\frac{1}{\sqrt{2}}(|0\rangle|+\rangle+|1\rangle|-\rangle) .
$$

The four virtual qubits at each site are projected down to a single physical qubit using the projector

$$
P=|0\rangle\langle 0000|+| 1\rangle\langle 1111| .
$$

The resulting state of the physical qubits on the lattice is the cluster state [8].

In our approach, we choose a nearest-neighbor twobody Ising-type Hamiltonian such as to effectively implement the above projection on four physical, rather than virtual, qubits. Consider a lattice of physical qubits with the same lattice structure as the virtual qubits above, as in Fig. 1. We label sites of four physical qubits by a Greek character, e.g., $\mu$, and define $S$ to be the set of all sites. Qubits at each site interact with nearest neighbors via a site Hamiltonian $H_{S}$ which is of Ising form

$$
H_{S}=-\sum_{\mu \in S} \sum_{i \sim i^{\prime}} \sigma_{(\mu, i)}^{z} \otimes \sigma_{\left(\mu, i^{\prime}\right)}^{z},
$$

where the first sum is over all sites, $i \sim i^{\prime}$ denotes pairs of neighboring qubits at site $\mu \in S$ connected by solid-line bonds in Fig. [1 and $\sigma_{(\mu, i)}^{z}$ is the Pauli $z$-operator for the physical qubit $i$ at site $\mu$.

The bonds between sites in Fig. 1 indicate a different two-body interaction, given by a two-body Hamiltonian of the form

$$
V=-\sum_{(\mu, i) \sim(\nu, j)}\left(\sigma_{(\mu, i)}^{z} \otimes \sigma_{(\nu, j)}^{x}+\sigma_{(\mu, i)}^{x} \otimes \sigma_{(\nu, j)}^{z}\right),
$$

where $\sigma^{x}$ is a physical Pauli $x$-operator, and $(\mu, i) \sim$ $(\nu, j)$ denotes pairs of neighboring qubits connected by dashed-line bonds in Fig. 1

The total Hamiltonian for the lattice is given by the sum of these terms, $H=g H_{S}+\lambda V$, where $g$ and $\lambda$ have units of energy. To investigate the spectrum of this Hamiltonian, we will use perturbation theory in $\lambda / g$, with $g H_{S}$ the unperturbed Hamiltonian.

We first investigate the spectrum of the unperturbed Hamiltonian $g H_{S}$. At a single site, there are three energy levels. The ground-state is degenerate, two-dimensional, and spanned by the states

$$
|0000\rangle \equiv\left|\mathbf{0}_{L}\right\rangle, \quad|1111\rangle \equiv\left|\mathbf{1}_{L}\right\rangle .
$$

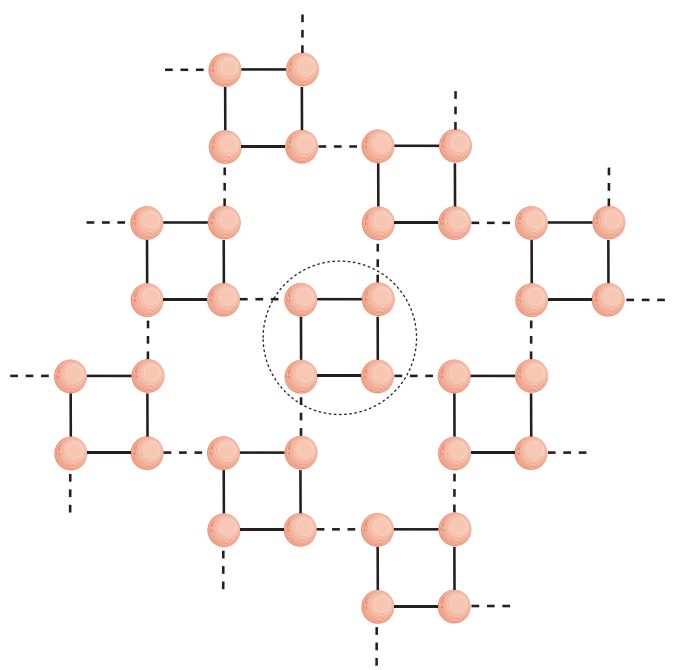

FIG. 1: Schematic of the spin lattice, with couplings. Solid lines represent an Ising-type interaction, as in the Hamiltonian of Eq. (3). The dashed lines represent a coupling as in the Hamiltonian of Eq. (4). Groups of four physical qubits bound by the Ising interaction, as within the circle, form a site - a logical qubit.

The ground state space of the unperturbed Hamiltonian, then, can be viewed as a logical qubit. The energy of this ground state space is $-4 g$. The first excited state is 12 -fold degenerate, and has a energy of 0 . The second excited state is 2-fold degenerate and has a energy of $4 g$.

Consider a lattice as in Fig. 10 consisting of $N_{S}$ sites with periodic boundary conditions. The spectrum of the unperturbed Hamiltonian $g H_{S}$ is straightforward. The ground-state space has energy $E_{0}^{(0)}=-4 g N_{S}$, is $2^{N_{S_{-}}}$ dimensional, and is spanned by all logical states of $N_{S}$ qubits. We denote this space $\mathcal{H}_{L}$. A convenient basis for this ground state space is as follows: let $|C\rangle$ denote the logical cluster state, and let $|C\{\alpha, \beta, \ldots\}\rangle$ denote the logical cluster state with logical $Z$-errors on the sites $\alpha, \beta, \ldots$. (The logical $Z$ operator is $Z \equiv\left|\mathbf{0}_{L}\right\rangle\left\langle\mathbf{0}_{L}|-| \mathbf{1}_{L}\right\rangle\left\langle\mathbf{1}_{L}\right|$.) The set of states $\{|C\rangle,|C\{\alpha\}\rangle,|C\{\alpha, \beta\}\rangle, \ldots\}$, running over logical $Z$-errors at all possible sites, forms a basis for this ground state space.

The first-excited space is $\left(12 N_{S} \cdot 2^{N_{S}-1}\right)$-dimensional, and has energy $E_{1}^{(0)}=-4 g\left(N_{S}-1\right)$. Thus, for the unperturbed Hamiltonian $g H_{S}$, the gap from the ground to first-excited space is $4 g$. The second-excited space has energy $E_{2}^{(0)}=-4 g\left(N_{S}-2\right)$. These energies will serve as the zeroth-order energies in perturbation theory for the total Hamiltonian.

We now turn to perturbation theory. The term $\lambda V$ in the Hamiltonian, representing the coupling on the bonds, breaks the degeneracy of the ground state space; however, as we will see, it only does so at a fourth-order perturbation in $\lambda / g$. Let $\Pi_{L}$ be the projection onto the ground state space of the unperturbed Hamiltonian $g H_{S}$, i.e., 
onto the "logical" space. It is straightforward to show that $\Pi_{L} V \Pi_{L}=0$, and thus there is no first-order correction to the energies. It is also straightforward to show that

$$
\Pi_{L} V^{2} \Pi_{L}=2 \times(\text { number of bonds })=4 N_{S} \Pi_{L} .
$$

(This constant arises from the fact that each Pauli term in $V$ squares to the identity.) Thus, there is a constant second-order correction to the ground-state energy - a shift - given by

$$
\lambda^{2} E_{0}^{(2)}=\frac{4 N_{S} \lambda^{2}}{E_{0}^{(0)}-E_{1}^{(0)}}=-N_{S} \frac{\lambda^{2}}{g} .
$$

There is no third-order correction to the ground state energy, following from the fact that $\Pi_{L} V^{3} \Pi_{L}=0$. Finally, at fourth-order, the degeneracy is broken. To calculate the correction to the energies, we will make use of two convenient properties of our encoding. First, we note that the product of all four $\sigma^{x}$ operators on a single site (one on each physical qubit) results in a logical $X$ operator

$$
X_{\mu}=\left|\mathbf{0}_{L}\right\rangle\left\langle\mathbf{1}_{L}|+| \mathbf{1}_{L}\right\rangle\left\langle\mathbf{0}_{L}\right|=\sigma_{(\mu, 1)}^{x} \sigma_{(\mu, 2)}^{x} \sigma_{(\mu, 3)}^{x} \sigma_{(\mu, 4)}^{x} .
$$

Second, we note that a single $\sigma^{z}$ operator acting on any of the four qubits at a site is equivalent to a logical $Z$ at that site. With these facts, we find that

$$
\Pi_{L} V^{4} \Pi_{L}-\left(\Pi_{L} V^{2} \Pi_{L}\right)^{2}=4 ! \sum_{\mu \in S} K_{\mu},
$$

where

$$
K_{\mu} \equiv X_{\mu} \prod_{\nu \sim \mu} Z_{\nu}
$$

is a stabilizer of the logical cluster state. In this expression, $X_{\mu}$ is a logical $X$ acting at the site $\mu$, and $Z_{\nu}$ is a logical $Z$ acting on a site that is connected to $\mu$ by a bond.

The operator $\Pi_{L} V^{4} \Pi_{L}$ is already diagonal in our chosen basis (the states of the form $|C\{\alpha, \beta, \ldots\}\rangle$ ). The fourth-order energy corrections for these states are determined by their eigenvalues, which are straightforward to calculate using the properties of stabilizers. First, we note that the cluster state $|C\rangle$ is an eigenstate of all stabilizers in the sum Eq. (9) with eigenvalue +1 , and thus the fourth-order correction for the energy associated with this state is

$$
\lambda^{4} E_{|C\rangle}^{(4)}=\frac{4 ! N_{S} \lambda^{4}}{\left(E_{0}^{(0)}-E_{1}^{(0)}\right)^{2}\left(E_{0}^{(0)}-E_{2}^{(0)}\right)}=-N_{S} \frac{3}{16} \frac{\lambda^{4}}{g^{3}} .
$$

Next, consider a state $|C\{\alpha\}\rangle=Z_{\alpha}|C\rangle$, a cluster state with a single $Z$-error at the site $\alpha$. This state is also an eigenstate of all stabilizers in the sum Eq. (9) with eigenvalue +1 except the stabilizer $K_{\alpha}$ which has eigenvalue
-1 . (This result is due to the fact that $X_{\alpha}$ and $Z_{\alpha}$ anticommute.) Thus, to fourth order, the spectrum of the Hamiltonian $H=g H_{S}+\lambda V$ is as follows: the component of the ground state in $\mathcal{H}_{L}$ is the cluster state $|C\rangle$, with energy

$$
E_{0}=-4 g N_{S}-N_{S} \frac{\lambda^{2}}{g}-N_{S} \frac{3}{16} \frac{\lambda^{4}}{g^{3}} .
$$

The $n$th excited space is $\left(\begin{array}{c}N_{S} \\ n\end{array}\right)$-dimensional. The component of this space in $\mathcal{H}_{L}$ is spanned by states obtained from $|C\rangle$ with $n$ logical $Z$ errors. These states have energy $E_{n}=E_{0}+n \Delta$, up to $n=N_{S}$, where

$$
\Delta \equiv E_{1}-E_{0}=\frac{3}{16} \frac{\lambda^{4}}{g^{3}} .
$$

(Above $n=N_{S}$, there is a large gap of order $6 g$ to the first manifold of illogical states, which are those that include a single physical $\sigma^{x}$ error.) We note that the gap $\Delta$ is independent of the size of the lattice. Intuitively, then, one may associate $\operatorname{logical} Z$ errors on any site with a fixed energy $\Delta=3 \lambda^{4} /\left(16 g^{3}\right)$ each.

Although the ground-state energy degeneracy of the unperturbed Hamiltonian $g H_{S}$ is not broken until fourth order, the lowest energy eigenstates are corrected even at first order as they will include terms from the higherenergy eigenspaces of $g H_{S}$. A simple counting of the states involved, and the magnitude of the components, will allow us to calculate the overlap of the first-ordercorrected ground state with the exact cluster state. It will also allow for a simple determination of an error probability per bond associated with this correction.

At first order, a state in the ground-state space (we will use $|C\rangle$ as an example, but this calculation holds for any state) will be corrected to include components from states $|\psi\rangle$ in the first excited space of $g H_{S}$ whenever $\langle\psi|V| C\rangle$ is non-zero. We note that every term of the form $\sigma^{x} \otimes \sigma^{z}$ or $\sigma^{z} \otimes \sigma^{x}$ from $V$ acting across a bond will connect $|C\rangle$ to a unique excited state, all of which form an orthogonal set; thus, summing over all the terms in $V$, there will be $4 N_{S}$ orthogonal states, denoted $|k\rangle$, for which $\langle k|V| C\rangle \neq 0$. The first order corrected ground state $\left|E_{0}\right\rangle$ is

$$
\left|E_{0}\right\rangle=\left(1+\frac{N_{S}}{4} \frac{\lambda^{2}}{g^{2}}\right)^{-1 / 2}\left(|C\rangle-\frac{\lambda}{4 g} \sum_{k=1}^{4 N_{S}}|k\rangle\right) .
$$

We now consider how measurement-based quantum computation can proceed using such a state. First, we need to verify that single qubit measurements alone suffice to perform universal quantum computation with an encoded cluster state. The logical cluster qubits consist of four physical qubits, and cluster-state computation requires making projective measurements on the logical qubits onto entangled superpositions of physical qubits the form $\left|\mathbf{0}_{L}\right\rangle \pm e^{-i \alpha}\left|\mathbf{1}_{L}\right\rangle$. By performing separate projective measurements on 3 of the 4 physical qubits in 
the $| \pm\rangle$ basis, the logical qubit is decoded into the state of the fourth physical qubit with at worst the addition of a known $Z$-error if an odd number of $|-\rangle$ outcomes are obtained 11 22. Such errors are easily compensated for by adapting future measurement bases in the manner standard for cluster state computing.

Next, consider the effect of the perturbative correction to the ground state of Eq. (14) in terms of its usefulness for quantum computation. Clearly, replacing the superposition of Eq. (14) with an incoherent mixture of the state $|C\rangle$ and the states $|k\rangle$ can only decrease the usefulness of the ground state for quantum computation. With this replacement, the resulting density matrix describing the ground state is a mixture of cluster states $|C\rangle$ with errors $\sigma^{x} \otimes \sigma^{z}$ (and $\sigma^{z} \otimes \sigma^{x}$ ) applied to all bonds independently with probability $p \simeq \lambda^{2} /(4 g)^{2}$. Note that this probability is independent of the size of the lattice, and thus these errors can be viewed as independent errors in the bonds between sites.

These errors take the system outside of the logical Hilbert space, because $\sigma^{x}$ on, say, the first physical qubit on a site maps logical states to states spanned by $|1000\rangle$ and $|0111\rangle$. However, these errors manifest themselves in the single-logical-qubit measurements described above as effective Pauli errors in the measurement. Thus, standard techniques for fault-tolerance in cluster state quantum computation [12, 13, 14] can be applied, provided the error probability $p \simeq \lambda^{2} /(4 g)^{2}$ is below some appropriate threshold.

Errors can also arise due to the finite energy gap, $\Delta=3 \lambda^{4} /\left(16 g^{3}\right)$. As we are using perturbation theory, we require $\lambda \ll g$ for the ground state to closely approximate the encoded cluster state. However, we emphasize that the energy scale $\lambda$ need only be small relative to $g$, and not in any absolute sense. The gap $\Delta$ is in turn small relative to $\lambda$. Consideration of the relative magnitudes of these energy scales, in conjunction with determining how cold such a lattice can be maintained, will determine whether this gap is sufficiently large to allow for quantum computation. Specifically, consider the effects of using a finite-temperature thermal state of this Hamiltonian. As investigated by 15], the thermal state will be a mixture of cluster states with logical $Z$-errors occurring independently at each site with probability $p=\left(1+\exp \left(\Delta / k_{B} T\right)\right)^{-1}$. The critical temperature above which the cluster state becomes too noisy to be useful for quantum computation scales as $k_{B} T_{\text {crit }} \sim \Delta$ [4, 15], which in this case is $k_{B} T_{\text {crit }} \sim(\lambda / g)^{3} \lambda$. Thus, the critical temperature is determined by the energy scale $\lambda$ and the order of perturbation theory at which the degeneracy is broken. (For the square lattice, the latter is four, leading to the $(\lambda / g)^{3}$ dependence.)

In relation to this point, we note that similar techniques to those presented here can be used to construct systems for any type of cluster state, not just on a square lattice, and a large number of other quantum states on a graph. This result follows directly from the origins of our method in the PEPS formalism. To generalize the above method, the number of physical qubits at each site must equal the number of bonds, i.e., the number of other sites that are directly connected to that site. For example, line- or ring-clusters can be created using only two physical qubits per site, a hexagonal lattice cluster state using three per site, and a cubic lattice cluster state using six per site. We note that the number of qubits per site determines the order in perturbation theory at which the ground-state degeneracy is broken. Continuing our argument from the previous paragraph, it is therefore interesting to consider the use of a hexagonal lattice cluster state of this form. Such a cluster state is a universal resource for quantum computation 16], and as the degeneracy is broken at third order, the energy gap (and thus the critical temperature) behaves as $\Delta_{\text {hex }} \sim \lambda^{3} / g^{2}$. This larger energy gap, as compared with the square lattice scaling of $\Delta_{\mathrm{sq}} \sim \lambda^{4} / g^{3}$, may make this state easier to prepare and maintain.

It is worthwhile to consider whether alternative local two-body Hamiltonians also yield an encoded cluster state as their ground state, or yield a non-cluster state that is nonetheless universal for quantum computation. For example, using a Heisenberg antiferromagnetic coupling on bonds and alternating sites between $Z Z$-type and $X X$-type Ising interactions leads to a similar result as above, where the ground state approximates an encoded cluster state. (To obtain this result, the choice of logical basis at each site must be alternated.) In the dynamical approach to cluster state creation, it has been shown that the Heisenberg interaction can be used instead of the Ising interaction [17], and logical encodings for this purpose have been investigated 18. A future line of research would be to investigate if lattices with entirely Heisenberg interactions (or other "natural" interactions on sites and bonds) can yield a computationallyuseful state as the ground state. We note that our lattice of Fig. 1] and the hexagonal lattice with three physical qubits per site, have identical structure to the "CaVOtype" lattices and star lattices, respectively, for which the Heisenberg antiferromagnetic coupling can lead to exotic quantum states [9, 19, 20].

We thank Chris Dawson, Andrew Doherty and Michael Varnava for helpful discussions and comments. SDB acknowledges support from the Australian Research Council. TR acknowledges support from the Engineering and Physical Sciences Research Council of the United Kingdom, and a University of Sydney Short-Term Visiting Fellowship.

[1] R. Raussendorf and H. J. Briegel, Phys. Rev. Lett. 86, 5188 (2001). 
[2] R. Raussendorf, D. E. Browne and H. J. Briegel, Phys. Rev. A 68, 022312 (2003), arXiv:quant-ph/0301052

[3] M. A. Nielsen, arXiv:quant-ph/0504097

[4] R. Raussendorf, S. Bravyi, J. Harrington, Phys. Rev. A 71, 062313 (2005), arXiv:quant-ph/0407255

[5] H. L. Haselgrove, M. A. Nielsen, and T. J. Osborne, Phys. Rev. Lett. 91, 210401 (2003), arXiv:quant-ph/0303022

[6] R. Oliveira and B. M. Terhal, arXiv:quant-ph/0504050

[7] J. Kempe, A. Kitaev, and O. Regev, SIAM Journal of Computing, 35, 1070 (2006), arXiv:quant-ph/0406180

[8] F. Verstraete and J. I. Cirac, Phys. Rev. A 70, 060302(R) (2004), arXiv:quant-ph/0311130

[9] J. Richter, J. Schulenburg, and A. Honecker, Lect. Notes Phys. 645, 85 (2004), arXiv:cond-mat/0412662

[10] J. Walgate, A. Short, L. Hardy and V. Vedral, Phys. Rev. Lett. 85, 4972 (2000), arXiv:quant-ph/0102073

[11] D. E. Browne and T. Rudolph, Phys. Rev. Lett. 95, 010501 (2005), arXiv:quant-ph/0405157.

[12] R. Raussendorf, PhD Thesis, Ludwig-Maximilians University, Munich (2003).

[13] M. A. Nielsen and C. M. Dawson, Phys. Rev. A 71, 042323 (2005), arXiv:quant-ph/0405134.

[14] P. Aliferis and D. W. Leung, Phys. Rev. A 73, 032308
(2006), arXiv:quant-ph/0503130

[15] A. Kay, J. K. Pachos, W. Dür, and H.-J. Briegel, New J. Phys. 8, 147 (2006), arXiv:quant-ph/0606177

[16] A. Van den Nest, A. Miyake, W. Dür, and H.J. Briegel, Phys. Rev. Lett. 97, 150504 (2006), arXiv:quant-ph/0604010

[17] M. Borhani and D. Loss, Phys. Rev. A 71, 034308 (2005), arXiv:quant-ph/0410145

[18] Y. S. Weinstein, C. S. Hellberg, and J. Levy, Phys. Rev. A 72, 020304(R) (2005), arXiv:quant-ph/0506032

[19] Z. Weihong, M. P. Gelfand, R. R. P. Singh, J. Oitmaa, and C. J. Hamer, Phys. Rev. B 55, 11377 (1997), arXiv:cond-mat/9603025

[20] J. Richter, J. Schulenburg, A. Honecker, and D. Schmalfuß, Phys. Rev. B 70, 174454 (2004), arXiv:cond-mat/0406103

[21] Using PEPS, it is standard to make use of the singlet state rather that the two-qubit cluster state. As both states are maximally-entangled and therefore related by local unitaries, these approaches are equivalent.

[22] The results of [10] prove that this result generalizes to any encoding. 